\title{
Efficacy of direct current stimulation on experimental sensory modalities and pain outcome measures in healthy participants: systematic review and meta-analysis.
}

\author{
Guillermo García-Barajas, PT, MsC ${ }^{1,2}$; Julian. Taylor, $\mathrm{PhD}^{1,6}$; Juan Pablo Romero Muñoz, MD, \\ PhD 5,6; Sergio Lerma Lara, PhD, MSc, PT 7,8; Alfonso Gil Martínez, PhD, MSc, PT 7,9,10; Josué \\ Fernández-Carnero, PT, PhD ${ }^{10,11,12}$.
}

1 Sensorimotor Function Group, Hospital Nacional de Parapléjicos, SESCAM, Toledo, Spain guille ce22@hotmail.es

2 Escuela Internacional de Doctorado. Department of Physical Therapy, Occupational

Therapy, Rehabilitation and Physical Medicine, Universidad Rey Juan Carlos, Alcorcón, 28933, Spain; guille_ce22@hotmail.es.

3 Biomechanical and Technical Aids Unit, Hospital Nacional de Parapléjicos, SESCAM, Toledo, Spain.

4 Harris Manchester College, University of Oxford, Oxford, United Kingdom.

juliantaylorgreen2@gmail.com

5 Facultad de Ciencias Experimentales. Universidad Francisco de Vitoria, Pozuelo de Alarcón, 28223, Spain; p.romero.prof@ufv.es

6 Brain Damage Unit, Beata María Ana Hospital, Madrid, 28007, Spain.

7 Department of Physical Therapy, Centro Superior de Estudios Universitarios La Salle, Universidad Autónoma de Madrid, Madrid, Spain. sergio.lerma@lasallecampus.es; alfonso.gil@lasallecampus.es

8 Motion in Brains Research Group, Institute of Neuroscience and Sciences of the Movement (INCIMOV),

Centro Superior de Estudios Universitarios La Salle, Universidad Autónoma de Madrid, Madrid, España. sergio.lerma@lasallecampus.es; alfonso.gil@lasallecampus.es

9 CranioSPain Research Group, Centro Superior de Estudios Universitarios La Salle, Universidad Autónoma de Madrid, Madrid, España. alfonso.gil@lasallecampus.es

10 Unit of Physiotherapy. La Paz Hospital Institute for Health Research, IdiPAZ, Madrid, Spain.

11 Department of Physical and Occupational Therapy, Rehabilitation and Physical Medicine, Universidad Rey Juan Carlos, Madrid, Spain. josue.fernandez@urjc.es

12 Grupo Multidisciplinar de Investigación y Tratamiento del Dolor, Grupo de Excelencia Investigadora, Universidad Rey Juan Carlos-Banco de Santander, Madrid, Spain.

Manuscript category: Systematic review.

Sources of financial support: This research was not funded by public or commercial organisations.

Authorship statement:

Conflicts of interest statement: None declared.

Corresponding Author: Fernández-Carnero Josué, PhD, MSc, PT; Faculty of Health Sciences. Physical Therapy, Occupational Therapy, Physical Medicine and Rehabilitation Department. Rey Juan Carlos University. Avenida de Atenas, s/n. Campus de Alcorcón, 28922 Alcorcón, Madrid, Spain. Phone: 0034- 914888949. Movile phone:0034-659706722 Fax: 0034- 914888957. E-mail: josue.fernandez@urjc.es

* Correspondence: sergio.lerma@lasallecampus.es; Tel.: +34-91 
Abstract: Background: Objectives. The objective of this study was to compare the efficacy of direct current stimulation (DCS) applied at the transcranial, suboccipital and spinal level on experimental sensory modalities and pain outcome measures in healthy subjects. The hypothesis of this study was that systematic analysis of the efficacy of DCS on modulating evoked thermal and mechanical pain modalities and mechanisms such as endogenous pain modulation in healthy individuals would reveal sensitive outcome measures help develop this technique for the control of chronic pain. Materials and Methods. Database searches were conducted up to December 2019 for randomized controlled trials that performed sham-controlled DCS of experimental sensory modalities and pain outcomes following transcranial, suboccipital and spinal locations in healthy participants. Standardized mean differences with $95 \%$ confidence intervals were calculated for sensory modalities, including random-effect metanalysis. Results: Thirty-one studies were included for analysis (647 participants). A significant decrease in pain intensity for active vs sham transcranial stimulation was identified for pain intensity ( $\mathrm{n}=158$; $\mathrm{SMD}=0.79 ; 95 \% \mathrm{CI}=0.56$ to 1.02$)$, a significant increase in heat pain threshold ( $\mathrm{n}=222 ; \mathrm{SMD}=1.16 ; 95 \% \mathrm{CI}=0.95$ to 1.37 ), and a significant increase in cold pain threshold ( $\mathrm{n}=155 ; \mathrm{SMD}=0.77,95 \%$ CI 0.53 to 1.01 ). No significant modulation of pressure pain threshold was identified with DCS and only a limited number of studies focused on experimental pain modulation following neuromodulation at the suboccipital or spinal level. Conclusions: These results show significant transcranial DCS neuromodulation of pain intensity and on thermal pain modalities. Future studies should focus on endogenous pain and sensory modality modulation with sham-controlled DCS applied at transcranial, suboccipital and spinal locations.

Keywords: Non-invasive direct current stimulation; Cortical, Suboccipital and Spinal stimulation; Quantitative sensory testing, Pain outcome measures, Endogenous pain modulation. 


\section{Introduction.}

Over the last few decades, direct current stimulation has been applied over the scalp as a non-invasive neuromodulation technique to modulate the brain excitability ${ }^{1,2}$. Indeed, direct current stimulation (DCS) provide a powerful tool for altering brain activity in a way that can outlast stimulation, and to influence neuronal function ${ }^{2}$. DCS techniques are used in several conditions to relieve pain such as fibromyalgia ${ }^{3}$, spinal cord injury ${ }^{4}$, migraine ${ }^{5}$, and chronic low back pain ${ }^{6}$. In studies published to date, DCS has been applied over transcranial level, with the aim of modulating excitability at cortical areas such as the primary motor $\operatorname{cortex}^{7}$ or the dorsolateral prefrontal cortex ${ }^{8}$, and subcortical areas such as the cerebellum ${ }^{9}$, and spinal cord ${ }^{10}$. It has been observed that could exist different mechanisms of action that depends on application modality, current parameters, and location of the DCS ${ }^{11,12}$.

Although transcranial direct current stimulation (tDCS) reduces pain intensity in people with different chronic pain 13,14 , the effect of this technique to reduce experimental pain in healthy volunteers is controversial ${ }^{15}, 16$. Furthermore, previous systematic reviews did not compare and evaluate the efficacy of pain modulation following DCS at subcortical sites such as the cortex, suboccipital and spinal level in healthy participants ${ }^{13,17}$. A systematic review and metanalysis of the DCS technique would clarify the efficacy of this technique to modulate experimental pain intensity ${ }^{18}$, pressure pain thresholds ${ }^{19}$, thermal pain thresholds ${ }^{20}$, endogenous pain modulatory mechanisms such as conditioned pain modulation ${ }^{21}$ and temporal summation magnitude ${ }^{22}$. The hypothesis of this study is that systematic analysis of the efficacy of DCS on modulating evoked thermal and mechanical pain modalities and mechanisms such as endogenous pain modulation in healthy individuals would reveal sensitive outcome measures to inform future research and clinical application of this stimulation technique.

The objective of this study is to compare the efficacy of direct current stimulation applied at the transcranial, suboccipital and spinal level on experimental sensory modalities and pain outcome measures in healthy subjects.

\section{Methods.}

A systematic review of the literature was performed following PRISMA guidelines ${ }^{23}$. A comprehensive review of computerized literature databases and searches to find unpublished trials were performed to minimize publication bias.

\subsection{Strategy of search and study selection.}

MEDLINE, accessed by PubMed (1990 to December 2019), EMBASE (1990 to December 2019), CINAHL (1990 to December 2019), Web of Science (1990 to December 2019), PsycInfo (1990 to December 2019), PEDro, and Google Scholar electronic databases were searched using the medical subject headings (MeSH): "transcranial direct current stimulation", "direct current stimulation", "pain", "healthy volunteers", "pain measurement", "cerebellum", “endogenous pain modulation", "nociception", "humans", "transcutaneous spinal direct current stimulation", "pain modulation", "quantitative sensory testing" as well as following free terms, combined in multiple search strategies with Boolean operators in order to find relevant articles: "spinal stimulation", "direct current stimulation", "sensory testing", "participants", "pain measurement", "clinical trials", "humans", "antinociception", "clinical study".

\subsection{Eligibility criteria.}

We followed the PICOS framework to organize the inclusion criteria. Population (P): study that included healthy subjects without pain in the last 3 months; Intervention (I): studies that use 
transcranial-DCS, spinal-DCS or cerebellar-DCS; Comparisons (C): interventional DCS or non-interventional DCS control; Outcomes $(\mathrm{O})$ : primary outcome that included quantitative sensory testing; and Study design (S): randomized controlled trials (RCTs) published in Spanish and English language.

Two authors screened the titles and abstracts of the initially identified studies to determine if they satisfied the selection criteria. Any disagreement was resolved through consensus. Full-text articles were retrieved for the selected titles, and reference lists of the retrieved articles were inspected to identify additional publications. The search was limited to studies written in English and Spanish. The search strategy described above yielded 84 results. Only articles reporting data on studies using the above-mentioned neurostimulation techniques in healthy subjects were considered eligible for inclusion. The inclusion criteria were: clinical trials, studies in humans, studies written in English and Spanish, studies that use transcranial-DCS, spinal-DCS or cerebellar-DCS as the intervention, studies in healthy volunteers, assessed variables like conditioned pain modulation, temporal summation magnitude and quantitative sensory threshold. The exclusion criteria were: studies that included participants with previous pain, studies that they did not include the current parameters, and studies that did not use quantitative sensory tests as variable of pain measurement. Thus, 31 studies were selected for analysis in this review, of these, 24 studies used transcranial-DCS, 6 studies used spinal-DCS and 2 studies used cerebellar-DCS (Figure 1).

\subsection{Data extraction.}

Physiotherapy Evidence Database (PEDro) scoring system was used for evaluating the selected studies ${ }^{24}$. Two authors independently screened the full-text articles to obtain a score in the PEDro scale. The PEDro tool consists of 11 questions with a maximum score of 10 . The following criteria were used for rating the methodological quality of a study: 9 to 10 , "excellent"; 6 to 8 , "good"; 4 to 5 , "fair"; and < 4 "poor" ${ }^{24}$. All studies were included in the analysis regardless of study quality.

Two independent reviewers (GGB and JFC) analysed the quality of all selected articles using the same methodology. Disagreements between reviewers were resolved by consensus by including a third reviewer. Inter-evaluator reliability was determined using the "kappa coefficient" ( $>0.7$ means high level of agreement among evaluators, 0.5-0.7 a moderate level of agreement, and $<0.5$ a low level of agreement) ${ }^{25}$. In each study, we extracted the following data to elaborate the characteristics of the table: (1) number of sessions and average age (2) current parameters (intensity, duration, current density, technique and electrode location), and (3) measurements (PT, CPM, and/or TS), (4) results.

\subsection{Risk of bias assessment.}

In the event of any discrepancies between the 2 reviewers, a consensus was attempted to be reached by discussion. If a full consensus could not be reached between the 2 reviewers after an exhaustive discussion, the opinion of a third reviewer was obtained, and the proceeding majority consensus was taken.

For the qualitative analysis of selected studies, the criteria for classification of evidence was followed for randomized controlled trials 26,27 . The evidence was classified into 5 levels based on methodological quality as follows: 1) "Strong", consistent findings among multiple high quality randomized controlled trials; 2) "Moderate", consistent findings among multiple low quality randomized controlled trials and/or controlled clinical trials, and/or one high quality randomized controlled trial; 3 ) "Limited", one low quality randomized controlled trial and/or controlled clinical trial; 4) "Conflicting", inconsistent findings among multiple trials (randomized controlled trials and/or controlled clinical trials); or 5) "No evidence from trials", no randomized controlled trials and/or controlled clinical trials ${ }^{26}$. 


\subsection{Data synthesis.}

Following the criteria proposed in the IMMPACT consensus statement ${ }^{28}$ and previous meta-analysis 29, we back transformed the standardized mean difference (SMD) to a mean difference using the mean standard deviation of the post-treatment sham group scores of the studies included in this analysis.

Data on each study's design, participant characteristics, intervention(s), outcome(s) and adverse event(s) were extracted from each of the selected studies. Pooled analyses were conducted for the primary outcome of pain intensity post-treatment and at follow-up. Treatment effect was reported as the SMD with 95\% confidence interval (CI) using the software of Meta-Analysis (Review Manager "Revman", version 5.4). The statistical significance of grouped SMDs was calculated as Hedges g, to consider the possible overestimation of the true effect magnitudes of sample in small studies. The magnitude of the "g" was interpreted according to the following scale ${ }^{30}:<0.20=$ insignificant effect, $0.20-0.49=$ small effect, $0.50-0.79=$ moderate effect, $\geq 0.80=$ great effect. Heterogeneity between studies was measured by the "I $\mathrm{I}^{2}$ statistic". An $\mathrm{I}^{2}$ value exceeding $50 \%$ was used as the threshold to identify significant statistical heterogeneity ${ }^{31,32}$.

The same inclusion criteria were used for the systematic review, but for inclusion in the meta-analysis, three more criteria were added: 1 ) the results of studies must have detailed information regarding the comparative statistical data (mean, standard deviation, and $95 \%$ confidence interval), 2) the data of analysed variables must be represented in at least two studies included; and 3) a minimum score of 6 points on the PEDro scale for clinical trial studies should be reached to allow their inclusion in the meta-analysis, because the inclusion of low methodological quality studies in a meta-analysis may overestimate the results obtained ${ }^{33}$.

\section{Results.}

Thirty-one studies met the inclusion criteria (Figure 1). Of the 31 studies included in this systematic review, 10 were scored as "excellent" and 21 were scored as "good" on the PEDro methodological quality scale (Table 1).

\subsection{Qualitative analysis.}

The mean age of subjects included in the review was $26.4 \pm 3.7$ years. The sample sizes of the included studies were small, between 10 and 41 subjects, except one study that included 75 subjects 34. Most of the included studies applied a single stimulation session, with the exception of two studies ${ }^{8,35}$ that performed 2 sessions; one study with 3 sessions ${ }^{36}, 4$ sessions ${ }^{37}$ and 5 sessions ${ }^{38}$. Table 2 shows the data of included studies.

Regarding the qualitative analysis of included studies a "strong" level of evidence was found for reduction of pain intensity with tDCS 34,39-42, a "limited" level of evidence in suboccipital (cerebellar) DCS ${ }^{43}$, and a "conflicting" level in spinal-DCS studies ${ }^{44}, 45$. For heat and cold pain thresholds, a "strong" level of evidence was found for increase of thermal thresholds in tDCS studies 10, 34, 38, 46-52. For pressure pain thresholds, a "conflicting" level of evidence was found for the increase of mechanical pain thresholds in tDCS studies $35,38,46,53,54$. A "limited" level of evidence was found for temporal summation magnitude in a spinal-DCS study ${ }^{45}$. For conditioned pain modulation a "moderate" level of evidence was found in tDCS studies ${ }^{35,42}$, and a "limited" level of evidence in spinal-DCS studies ${ }^{36}$. Table 3 shows the qualitative data of included studies.

\subsection{Quantitative analysis.}


For the quantitative analysis of pain intensity modulation with DCS, five studies were included ${ }^{34}$ $40-42,53$, with the meta-analysis of these studies showing statistically significant differences for the decrease of pain intensity for active compared to control tDCS ( $\mathrm{n}=158$; SMD $=0.79,95 \%$ CI 0.56 to 1.02; Figure 2). Heterogeneity findings were found among the groups $\left(\mathrm{Chi}^{2}=10.62 ; \mathrm{df}=4 ; \mathrm{I}^{2}=62 \% ; \mathrm{P}\right.$ $=0.03)$.

Five studies of pressure pain threshold modulation were included in the review $35,38,46,53,54$, with the meta-analysis failing to show statistically significant differences for the increase of mechanical thresholds for active vs control tDCS ( $\mathrm{n}=82$; SMD $=0.06,95 \%$ CI -0.25 to 0.36 ; Figure 3). Homogeneity findings were found in the groups $\left(\mathrm{Chi}^{2}=0.44 ; \mathrm{df}=4 ; \mathrm{I}^{2}=0 \% ; \mathrm{P}=0.98\right)$.

Eight studies of heat pain modulation with DCS were included for analysis 34, 38, 46-49, 51, 53 showing statistically significant differences for active vs control tDCS ( $\mathrm{n}=222$; $\mathrm{SMD}=1.16,95 \%$ CI 0.95 to 1.37 ; Figure 4). Heterogeneity findings were found in the groups $\left(\mathrm{Chi}^{2}=71.47 ; \mathrm{df}=7 ; \mathrm{I}^{2}=90 \% ; \mathrm{P}<0.01\right)$.

Eight studies of cold pain modulation with DCS were included for analysis 8, 10,38,46-48,50,52, with the meta-analysis of the studies showing statistically significant differences for active vs control tDCS (n $=155 ; \mathrm{SMD}=0.77,95 \% \mathrm{CI} 0.53$ to 1.01 ; Figure 5). Heterogeneity findings were found in the groups $\left(\mathrm{Chi}^{2}=31.74 ; \mathrm{df}=7 ; \mathrm{I}^{2}=78 \% ; \mathrm{P}<0.01\right)$.

\subsection{Adverse and side effects.}

Of 31 studies included in this systematic review, 15 registered adverse or side effects were related to DCS interventions 8, 9, 15, 20, 35, 38, 39, 41-43, 45, 47, 49, 52, 54. Furthermore, 5 studies registered tingling and itching sensations during the interventions with DCS $8,39,45,52,54$. 


\section{Discussion.}

This systematic review and metanalysis revealed significant DCS neuromodulation of pain intensity and on thermal pain modalities following transcranial cortical application. The effect size for modulation of pain intensity and thermal pain thresholds was large. However only a limited number of studies were found for DCS neuromodulation of experimental pain was found following application at the suboccipital or spinal level. No significant modulation of pressure pain threshold was found with DCS technic and only a limited number of studies focused on experimental pain modulation following neuromodulation at the suboccipital or spinal level.

\subsection{The effects of DCS on experimental pain intensity, temporal summation and conditioned pain modulation.}

The meta-analysis of selected studies showed that active tDCS was effective in reducing pain intensity (Figure 2). Of 31 studies included, only 5 were selected for analysis, with four studies showing a significant reduction in pain intensity following active tDCS compared to sham tDCS ${ }^{34}$, 40-42, and one study finding no significant difference between active and sham $\mathrm{tDCS}{ }^{53}$. A previous systematic review found similar results regarding pain intensity in healthy volunteers ${ }^{37}$. Furthermore, our results support the findings of other meta-analyses studies that found a reduction in pain intensity using tDCS in chronic pain conditions like fibromyalgia 55 , spinal cord injury ${ }^{56}$, and chronic orofacial pain ${ }^{57}$.

Qualitative analysis of the selected studies found a "strong" level of evidence for the reduction of pain intensity in transcranial-DCS studies ${ }^{34,39-42}$, a "limited" level of evidence in cerebellar-DCS studies ${ }^{43}$, and a "conflicting" level of evidence in spinal-DCS studies ${ }^{44,45}$. These results were similar to previous DCS systematic reviews regarding the level of evidence for pain intensity modulation with tDCS in people with chronic pain conditions 13,58. A "moderate" level of evidence was found for a reduction in temporal summation in transcranial-DCS studies 47,53,59, and a "moderate" level of evidence for conditioned pain modulation ${ }^{35,42}$. However, a meta-analysis of the effect of tDCS on temporal summation and conditioned pain modulation was not possible as only a limited number of studies were found. Previous systematic reviews have found that modulation of both these pain outcome measures in healthy populations 13,17 , although the application of DCS was combined with other interventions like therapeutic exercise or analgesic medications.

\subsection{The effect of DCS on pressure pain thresholds.}

The meta-analysis of selected studies did not demonstrate that active tDCS was efficacious for increasing the PPTs (Figure 3). Of the 31 studies selected, only 5 were selected for quantitative analysis, with four studies failing to demonstrate a significant increase of PPT following active tDCS compared to sham $\operatorname{tDCS} 38,46,53,54$, and with only one study showing a significant increase following tDCS group ${ }^{35}$. These results do not support the findings of a previous systematic review about the effects of tDCS in chronic pain patients ${ }^{60}$. Furthermore, in the present systematic review a "conflicting" level of evidence was found for mechanical pain threshold modulation in tDCS studies $35,38,46,53,54$.

\subsection{The effects of DCS on heat and cold pain thresholds.}

The meta-analysis of selected studies demonstrate that active tDCS was efficacious in increasing cold and heat pain thresholds (Figure 4 y 5). Of the 31 studies included, 12 were selected for quantitative analysis of heat and cold pain thresholds following active tDCS, with six studies showing a significant increase in heat pain threshold in active tDCS groups in front of sham tDCS groups $34,38,46,47,49,51$, and two studies failing to demonstrate a significant difference between active and sham tDCS 48,53 . For cold pain threshold, seven studies found a significant increase in threshold following active tDCS compared to sham tDCS 8, 10,38,46,47,50,52, and only one study failed to show a 
significant difference between active and sham tDCS ${ }^{48}$. The majority of published studies support DCS modulation of thermal detection or pain thresholds $34,46,47,50$ or tolerance in healthy participants $8,51,52$. The results obtained in this study are supported by a recent systematic review that found an improvement in thermal pain thresholds ${ }^{17}$.

Although the mechanisms are not known by which tDCS selectively modulate thermal pain measures compared to mechanical pain, several pain processing centres have been shown to modulate thermal sensation including the hypothalamic nuclei, preoptic area, amygdala ${ }^{61,62}$ and thalamus ${ }^{63}$, some of which have been shown to constitute part of the endogenous pain modulation network ${ }^{64}$.

\subsection{Limitations and future directions.}

According to the Cochrane Handbook for Systematic Reviews, "potential advantages of meta-analysis include an increase in power, an improvement in precision, the ability to answer questions not posted by individual studies, and the opportunity to settle controversies arising from conflicting claims". That is, establishing clear methods and specific criteria can minimise the bias that the reviewer brings to the study. However, we cannot limit the bias that is within the literature about DCS ${ }^{16}$. The present systematic review was limited to English and Spanish language studies, and most of included studies used small samples that influence in effect sizes and therefore might affect pooled results in the meta-analyses. The major limitation of this systematic review is the lack of DCS studies applied at the subcortical level and spinal level, which would influence the effect size. These studies were not included in the meta-analyses. Another important limitation in the current review is the heterogeneity found in the DCS protocols, with several factors related to current intensity, size of the DCS electrodes, duration of the interventions and the number of sessions ${ }^{13,58}$. Furthermore, most of included studies did not perform blinding to clinicians and participants, and only five included studies carried out more than one session with DCS.

Moving one step beyond the state of the art, the next step for DCS is in the clinical field. A translational research application of the main findings supported in the literature could be the inspiring trigger for new research and the identification of the barriers that are contributing factors for solving the gap between the academic field and the clinical practice.

\section{Conclusions.}

These results show significant transcranial DCS neuromodulation of pain intensity and on thermal pain modalities. Future clinical studies with patients should focus on endogenous pain and sensory modality modulation with sham-controlled DCS applied at transcranial, suboccipital and spinal locations.

Author Contributions: Conceptualization, X.X. and Y.Y.; methodology, X.X.; software, X.X.; validation, X.X., Y.Y. and Z.Z.; formal analysis, X.X.; investigation, X.X.; resources, X.X.; data curation, X.X.; writing - original draft preparation, X.X.; writing-review and editing, X.X.; visualization, X.X.; supervision, X.X.; project administration, X.X.; funding acquisition, Y.Y. All authors have read and agreed to the published version of the manuscript.

Funding: This research received no external funding, neither has an external sponsor.

\section{Acknowledgments:}

Conflicts of Interest: The authors declare no conflict of interest. 


\section{References}

1. Nitsche, M. A.; Cohen, L. G.; Wassermann, E. M.; Priori, A.; Lang, N.; Antal, A.; Paulus, W.; Hummel, F.; Boggio, P. S.; Fregni, F.; Pascual-Leone, A., Transcranial direct current stimulation: State of the art 2008. Brain Stimul 2008, 1 (3), 206-23.

2. Nitsche, M. A.; Paulus, W., Excitability changes induced in the human motor cortex by weak transcranial direct current stimulation. J Physiol 2000, 527 Pt 3, 633-9.

3. Fregni, F.; Gimenes, R.; Valle, A. C.; Ferreira, M. J.; Rocha, R. R.; Natalle, L.; Bravo, R.; Rigonatti, S. P.; Freedman, S. D.; Nitsche, M. A.; Pascual-Leone, A.; Boggio, P. S., A randomized, sham-controlled, proof of principle study of transcranial direct current stimulation for the treatment of pain in fibromyalgia. Arthritis Rheum 2006, 54 (12), 3988-98.

4. Ngernyam, N.; Jensen, M. P.; Arayawichanon, P.; Auvichayapat, N.; Tiamkao, S.; Janjarasjitt, S.; Punjaruk, W.; Amatachaya, A.; Aree-uea, B.; Auvichayapat, P., The effects of transcranial direct current stimulation in patients with neuropathic pain from spinal cord injury. Clin Neurophysiol 2015, 126 (2), 382-90.

5. Dasilva, A. F.; Mendonca, M. E.; Zaghi, S.; Lopes, M.; Dossantos, M. F.; Spierings, E. L.; Bajwa, Z.; Datta, A.; Bikson, M.; Fregni, F., tDCS-induced analgesia and electrical fields in pain-related neural networks in chronic migraine. Headache 2012, 52 (8), 1283-95.

6. Mariano, T. Y.; Burgess, F. W.; Bowker, M.; Kirschner, J.; Van't Wout-Frank, M.; Jones, R. N.; Halladay, C. W.; Stein, M.; Greenberg, B. D., Transcranial Direct Current Stimulation for Affective Symptoms and Functioning in Chronic Low Back Pain: A Pilot Double-Blinded, Randomized, Placebo-Controlled Trial. Pain Med 2019, 20 (6), 1166-1177.

7. Hughes, S.; Grimsey, S.; Strutton, P. H., Primary Motor Cortex Transcranial Direct Current Stimulation Modulates Temporal Summation of the Nociceptive Withdrawal Reflex in Healthy Subjects. Pain Med 2019, 20 (6), 1156-1165.

8. Mariano, T. Y.; Van't Wout, M.; Garnaat, S. L.; Rasmussen, S. A.; Greenberg, B. D., Transcranial Direct Current Stimulation (tDCS) Targeting Left Dorsolateral Prefrontal Cortex Modulates Task-Induced Acute Pain in Healthy Volunteers. Pain Med 2016, 17 (4), 737-45.

9. Pereira, M.; Rafiq, B.; Chowdhury, E.; Babayev, J.; Boo, H.; Metwaly, R.; Sandilya, P.; Chusid, E.; Battaglia, F., Anodal cerebellar tDCS modulates lower extremity pain perception. NeuroRehabilitation 2017, 40 (2), 195-200.

10. Truini, A.; Vergari, M.; Biasiotta, A.; La Cesa, S.; Gabriele, M.; Di Stefano, G.; Cambieri, C.; Cruccu, G.; Inghilleri, M.; Priori, A., Transcutaneous spinal direct current stimulation inhibits nociceptive spinal pathway conduction and increases pain tolerance in humans. Eur J Pain 2011, 15 (10), 1023-7.

11. Antal, A.; Alekseichuk, I.; Bikson, M.; Brockmoller, J.; Brunoni, A. R.; Chen, R.; Cohen, L. G.; Dowthwaite, G.; Ellrich, J.; Floel, A.; Fregni, F.; George, M. S.; Hamilton, R.; Haueisen, J.; Herrmann, C. S.; Hummel, F. C.; Lefaucheur, J. P.; Liebetanz, D.; Loo, C. K.; McCaig, C. D.; Miniussi, C.; Miranda, P. C.; Moliadze, V.; Nitsche, M. A.; Nowak, R.; Padberg, F.; Pascual-Leone, A.; Poppendieck, W.; Priori, A.; Rossi, S.; Rossini, P. M.; Rothwell, J.; Rueger, M. A.; Ruffini, G.; Schellhorn, K.; Siebner, H. R.; Ugawa, Y.; Wexler, A.; Ziemann, U.; Hallett, M.; Paulus, W., Low intensity transcranial electric stimulation: Safety, ethical, legal regulatory and application guidelines. Clin Neurophysiol 2017, 128 (9), 1774-1809.

12. Ridding, M. C.; Ziemann, U., Determinants of the induction of cortical plasticity by non-invasive brain stimulation in healthy subjects. J Physiol 2010, 588 (Pt 13), 2291-304. 
13. O'Connell, N. E.; Marston, L.; Spencer, S.; DeSouza, L. H.; Wand, B. M., Non-invasive brain stimulation techniques for chronic pain. Cochrane Database Syst Rev 2018, 4, Cd008208.

14. Pinto, C. B.; Teixeira Costa, B.; Duarte, D.; Fregni, F., Transcranial Direct Current Stimulation as a Therapeutic Tool for Chronic Pain. J ect 2018, 34 (3), e36-e50.

15. Ihle, K.; Rodriguez-Raecke, R.; Luedtke, K.; May, A., tDCS modulates cortical nociceptive processing but has little to no impact on pain perception. Pain 2014, 155 (10), 2080-7.

16. O'Connell, N. E.; Cossar, J.; Marston, L.; Wand, B. M.; Bunce, D.; Moseley, G. L.; De Souza, L. H., Rethinking clinical trials of transcranial direct current stimulation: participant and assessor blinding is inadequate at intensities of 2mA. PloS one 2012, 7 (10), e47514.

17. Giannoni-Luza, S.; Pacheco-Barrios, K.; Cardenas-Rojas, A.; Mejia-Pando, P. F.; Luna-Cuadros, M. A.; Barouh, J. L.; Gnoatto-Medeiros, M.; Candido-Santos, L.; Barra, A.; Caumo, W.; Fregni, F., Non-invasive motor cortex stimulation effects on quantitative sensory testing (QST) in healthy and chronic pain subjects: a systematic review and meta-analysis. Pain 2020.

18. Heller, G. Z.; Manuguerra, M.; Chow, R., How to analyze the Visual Analogue Scale: Myths, truths and clinical relevance. Scand J Pain 2016, 13, 67-75.

19. Treede, R. D.; Rolke, R.; Andrews, K.; Magerl, W., Pain elicited by blunt pressure: neurobiological basis and clinical relevance. Pain 2002, 98 (3), 235-40.

20. Moloney, N. A.; Hall, T. M.; Doody, C. M., Reliability of thermal quantitative sensory testing: a systematic review. J Rehabil Res Dev 2012, 49 (2), 191-207.

21. Yarnitsky, D.; Arendt-Nielsen, L.; Bouhassira, D.; Edwards, R. R.; Fillingim, R. B.; Granot, M.; Hansson, P.; Lautenbacher, S.; Marchand, S.; Wilder-Smith, O., Recommendations on terminology and practice of psychophysical DNIC testing. Eur J Pain 2010, 14 (4), 339.

22. Staud, R.; Weyl, E. E.; Riley, J. L., 3rd; Fillingim, R. B., Slow temporal summation of pain for assessment of central pain sensitivity and clinical pain of fibromyalgia patients. PloS one 2014, 9 (2), e89086.

23. Moher, D.; Liberati, A.; Tetzlaff, J.; Altman, D. G., Preferred reporting items for systematic reviews and meta-analyses: the PRISMA statement. PLoS Med 2009, 6 (7), e1000097.

24. Moseley, A. M.; Herbert, R. D.; Sherrington, C.; Maher, C. G., Evidence for physiotherapy practice: a survey of the Physiotherapy Evidence Database (PEDro). Aust J Physiother 2002, 48 (1), 43-9.

25. Becker, G., Creating comparability among reliability coefficients: the case of Cronbach alpha and Cohen kappa. Psychol Rep 2000, 87 (3 Pt 2), 1171-82.

26. Furlan, A. D.; Pennick, V.; Bombardier, C.; van Tulder, M., 2009 updated method guidelines for systematic reviews in the Cochrane Back Review Group. Spine (Phila Pa 1976) 2009, 34 (18), 1929-41.

27. van Tulder, M.; Furlan, A.; Bombardier, C.; Bouter, L., Updated method guidelines for systematic reviews in the cochrane collaboration back review group. Spine (Phila Pa 1976) 2003, 28 (12), 1290-9.

28. Liberati, A.; Altman, D. G.; Tetzlaff, J.; Mulrow, C.; Gotzsche, P. C.; Ioannidis, J. P.; Clarke, M.; Devereaux, P. J.; Kleijnen, J.; Moher, D., The PRISMA statement for reporting systematic reviews and meta-analyses of studies that evaluate health care interventions: explanation and elaboration. PLoS Med 2009, 6 (7), e1000100.

29. Tricco, A. C.; Lillie, E.; Zarin, W.; O'Brien, K. K.; Colquhoun, H.; Levac, D.; Moher, D.; Peters, M. D. J.; Horsley, T.; Weeks, L.; Hempel, S.; Akl, E. A.; Chang, C.; McGowan, J.; Stewart, L.; Hartling, L.; Aldcroft, A.; Wilson, M. G.; Garritty, C.; Lewin, S.; Godfrey, C. M.; Macdonald, M. T.; Langlois, E. V.; Soares-Weiser, K.; Moriarty, J.; Clifford, T.; Tuncalp, O.; Straus, S. E., PRISMA Extension for Scoping Reviews (PRISMA-ScR): Checklist and Explanation. Ann Intern Med 2018, 169 (7), 467-473. 
30. Cohen, J., A power primer. Psychol Bull 1992, 112 (1), 155-9.

31. Higgins, J. P.; Thompson, S. G.; Deeks, J. J.; Altman, D. G., Measuring inconsistency in meta-analyses. Bmj 2003, 327 (7414), 557-60.

32. Huedo-Medina, T. B.; Sanchez-Meca, J.; Marin-Martinez, F.; Botella, J., Assessing heterogeneity in meta-analysis: Q statistic or 12 index? Psychol Methods 2006, 11 (2), 193-206.

33. Moher, D.; Pham, B.; Jones, A.; Cook, D. J.; Jadad, A. R.; Moher, M.; Tugwell, P.; Klassen, T. P., Does quality of reports of randomised trials affect estimates of intervention efficacy reported in meta-analyses? Lancet 1998, 352 (9128), 609-13.

34. Aslaksen, P. M.; Vasylenko, O.; Fagerlund, A. J., The effect of transcranial direct current stimulation on experimentally induced heat pain. Exp Brain Res 2014, 232 (6), 1865-73.

35. Flood, A.; Waddington, G.; Cathcart, S., High-definition transcranial direct current stimulation enhances conditioned pain modulation in healthy volunteers: a randomized trial. The Journal of Pain 2016, 17 (5), $600-605$.

36. Perrotta, A.; Bolla, M.; Anastasio, M. G.; Serrao, M.; Sandrini, G.; Pierelli, F., Modulation of temporal summation threshold of the nociceptive withdrawal reflex by transcutaneous spinal direct current stimulation in humans. Clin Neurophysiol 2016, 127 (1), 755-761.

37. Vaseghi, B.; Zoghi, M.; Jaberzadeh, S., Does anodal transcranial direct current stimulation modulate sensory perception and pain? A meta-analysis study. Clin Neurophysiol 2014, 125 (9), 1847-58.

38. Moloney, T. M.; Witney, A. G., Pressure pain thresholds increase after preconditioning $1 \mathrm{~Hz}$ repetitive transcranial magnetic stimulation with transcranial direct current stimulation. PloS one 2014, 9 (3), e92540.

39. Hamner, J. W.; Villamar, M. F.; Fregni, F.; Taylor, J. A., Transcranial direct current stimulation (tDCS) and the cardiovascular responses to acute pain in humans. Clin Neurophysiol 2015, 126 (5), 1039-46.

40. Henriques, I. A. D.; Lattari, E.; Torres, G.; Rodrigues, G. M.; Oliveira, B. R. R.; Neto, G. A. M.; Neto, S. R. M.; Machado, S., Can transcranial direct current stimulation improve range of motion and modulate pain perception in healthy individuals? Neurosci Lett 2019, 707, 134311.

41. Naylor, J. C.; Borckardt, J. J.; Marx, C. E.; Hamer, R. M.; Fredrich, S.; Reeves, S. T.; George, M. S., Cathodal and anodal left prefrontal tDCS and the perception of control over pain. Clin J Pain 2014, 30 (8), 693-700.

42. Reidler, J. S.; Mendonca, M. E.; Santana, M. B.; Wang, X.; Lenkinski, R.; Motta, A. F.; Marchand, S.; Latif, L.; Fregni, F., Effects of motor cortex modulation and descending inhibitory systems on pain thresholds in healthy subjects. J Pain 2012, 13 (5), 450-8.

43. Bocci, T.; Marceglia, S.; Vergari, M.; Cognetto, V.; Cogiamanian, F.; Sartucci, F.; Priori, A., Transcutaneous spinal direct current stimulation modulates human corticospinal system excitability. $J$ Neurophysiol 2015, 114 (1), 440-6.

44. Lenoir, C.; Jankovski, A.; Mouraux, A., Anodal Transcutaneous Spinal Direct Current Stimulation (tsDCS) Selectively Inhibits the Synaptic Efficacy of Nociceptive Transmission at Spinal Cord Level. Neuroscience 2018, 393, 150-163.

45. Schweizer, L. M.; Zahn, P. K.; Pogatzki-Zahn, E. M.; Magerl, W.; Tegenthoff, M.; Meyer-Friessem, C. H., Influence of transcutaneous spinal stimulation on human LTP-like pain amplification. A randomized, double-blind study in volunteers. Clin Neurophysiol 2017, 128 (8), 1413-1420.

46. Bachmann, C. G.; Muschinsky, S.; Nitsche, M. A.; Rolke, R.; Magerl, W.; Treede, R. D.; Paulus, W.; Happe, S., Transcranial direct current stimulation of the motor cortex induces distinct changes in thermal and mechanical sensory percepts. Clin Neurophysiol 2010, 121 (12), 2083-9. 
47. Borckardt, J. J.; Bikson, M.; Frohman, H.; Reeves, S. T.; Datta, A.; Bansal, V.; Madan, A.; Barth, K.; George, M. S., A pilot study of the tolerability and effects of high-definition transcranial direct current stimulation (HD-tDCS) on pain perception. J Pain 2012, 13 (2), 112-20.

48. Grundmann, L.; Rolke, R.; Nitsche, M. A.; Pavlakovic, G.; Happe, S.; Treede, R. D.; Paulus, W.; Bachmann, C. G., Effects of transcranial direct current stimulation of the primary sensory cortex on somatosensory perception. Brain Stimul 2011, 4 (4), 253-60.

49. Meeker, T. J.; Keaser, M. L.; Khan, S. A.; Gullapalli, R. P.; Seminowicz, D. A.; Greenspan, J. D., Non-invasive Motor Cortex Neuromodulation Reduces Secondary Hyperalgesia and Enhances Activation of the Descending Pain Modulatory Network. Front Neurosci 2019, 13, 467.

50. Mordillo-Mateos, L.; Dileone, M.; Soto-Leon, V.; Brocalero-Camacho, A.; Perez-Borrego, Y. A.; Onate-Figuerez, A.; Aguilar, J.; Oliviero, A., Effects of transcranial direct current stimulation on temperature and pain perception. Sci Rep 2017, 7 (1), 2946.

51. Mylius, V.; Jung, M.; Menzler, K.; Haag, A.; Khader, P. H.; Oertel, W. H.; Rosenow, F.; Lefaucheur, J. P., Effects of transcranial direct current stimulation on pain perception and working memory. Eur J Pain 2012, 16 (7), 974-82.

52. Zandieh, A.; Parhizgar, S. E.; Fakhri, M.; Taghvaei, M.; Miri, S.; Shahbabaie, A.; Esteghamati, S.; Ekhtiari, H., Modulation of cold pain perception by transcranial direct current stimulation in healthy individuals. Neuromodulation 2013, 16 (4), 345-8; discussion 348.

53. Jurgens, T. P.; Schulte, A.; Klein, T.; May, A., Transcranial direct current stimulation does neither modulate results of a quantitative sensory testing protocol nor ratings of suprathreshold heat stimuli in healthy volunteers. Eur J Pain 2012, 16 (9), 1251-63.

54. Vaseghi, B.; Zoghi, M.; Jaberzadeh, S., Differential effects of cathodal transcranial direct current stimulation of prefrontal, motor and somatosensory cortices on cortical excitability and pain perception - a double-blind randomised sham-controlled study. Eur J Neurosci 2015, 42 (7), 2426-37.

55. Zhu, C. E.; Yu, B.; Zhang, W.; Chen, W. H.; Qi, Q.; Miao, Y., Effiectiveness and safety of transcranial direct current stimulation in fibromyalgia: A systematic review and meta-analysis. J Rehabil Med 2017, 49 (1), 2-9. 56. Mehta, S.; McIntyre, A.; Guy, S.; Teasell, R. W.; Loh, E., Effectiveness of transcranial direct current stimulation for the management of neuropathic pain after spinal cord injury: a meta-analysis. Spinal Cord 2015, 53 (11), 780-5.

57. Herrero Babiloni, A.; Guay, S.; Nixdorf, D. R.; de Beaumont, L.; Lavigne, G., Non-invasive brain stimulation in chronic orofacial pain: a systematic review. J Pain Res 2018, 11, 1445-1457.

58. Lefaucheur, J. P.; Antal, A.; Ayache, S. S.; Benninger, D. H.; Brunelin, J.; Cogiamanian, F.; Cotelli, M.; De Ridder, D.; Ferrucci, R.; Langguth, B.; Marangolo, P.; Mylius, V.; Nitsche, M. A.; Padberg, F.; Palm, U.; Poulet, E.; Priori, A.; Rossi, S.; Schecklmann, M.; Vanneste, S.; Ziemann, U.; Garcia-Larrea, L.; Paulus, W., Evidence-based guidelines on the therapeutic use of transcranial direct current stimulation (tDCS). Clin Neurophysiol 2017, 128 (1), 56-92.

59. Hughes, S. W.; Ali, M.; Sharma, P.; Insan, N.; Strutton, P. H., Frequency-dependent top-down modulation of temporal summation by anodal transcranial direct-current stimulation of the primary motor cortex in healthy adults. Eur J Pain 2018.

60. Ahn, H.; Suchting, R.; Woods, A. J.; Miao, H.; Green, C.; Cho, R. Y.; Choi, E.; Fillingim, R. B., Bayesian analysis of the effect of transcranial direct current stimulation on experimental pain sensitivity in older adults with knee osteoarthritis: randomized sham-controlled pilot clinical study. J Pain Res 2018, 11, 2071-2082. 
61. Newman, H. M.; Stevens, R. T.; Apkarian, A. V., Direct spinal projections to limbic and striatal areas: anterograde transport studies from the upper cervical spinal cord and the cervical enlargement in squirrel monkey and rat. J Comp Neurol 1996, 365 (4), 640-58.

62. Clewett, D.; Schoeke, A.; Mather, M., Amygdala functional connectivity is reduced after the cold pressor task. Cogn Affect Behav Neurosci 2013, 13 (3), 501-18.

63. Smith, M. V.; Apkarian, A. V.; Hodge, C. J., Jr., Somatosensory response properties of contralaterally projecting spinothalamic and nonspinothalamic neurons in the second cervical segment of the cat. J Neurophysiol 1991, 66 (1), 83-102.

64. Sagalajev, B.; Wei, H.; Chen, Z.; Albayrak, I.; Koivisto, A.; Pertovaara, A., Oxidative Stress in the Amygdala Contributes to Neuropathic Pain. Neuroscience 2018, 387, 92-103. 